\title{
Extrato metanólico de folhas de mandioca como alternativa ao controle da lagarta-do-cartucho e de formigas cortadeiras
}

\section{Cassava leaf methanolic extract as an alternative to control of fall armyworm and leaf cutter ants}

\author{
Mírian Aparecida Isidro Santos ${ }^{1 *}$; Angelita Duarte Corrêa ${ }^{2 *}$; \\ Ana Paula de Carvalho Alves ${ }^{1}$; Anderson Assaid Simão ${ }^{1}$; Dejane Santos Alves ${ }^{3}$; \\ Rodrigo Lopes de Oliveira ${ }^{4}$; Adelir Aparecida Saczk²; Geraldo Andrade Carvalho²
}

\section{Resumo}

Objetivou-se neste trabalho caracterizar os compostos fenólicos e avaliar o efeito, em condições de laboratório, do extrato metanólico do pó de folhas de mandioca sobre o desenvolvimento da lagarta-docartucho $S$. frugiperda e da saúva-limão Atta sexdens rubropilosa. O referido extrato, nas concentrações de $250,500,1000$ e $1500 \mathrm{mg} \mathrm{kg}^{-1}$, foi incorporado na dieta artificial, a qual foi exposta a lagarta para avaliar características biológicas. Logo após a emergência dos insetos, outro experimento foi realizado para verificar a possível atividade subletal do extrato, para isso casais de $S$. frugiperda foram isolados em gaiolas e as posturas foram recolhidas para a quantificação. O extrato de Manihot esculenta Crantz provocou redução na porcentagem de sobrevivência das lagartas, bem como no número de ovos. A seguir o mesmo extrato foi solubilizado em etanol 10\% e aplicado em formigas, observou-se a mortalidade em comparação com a testemunha. Conclui-se que o extrato metanólico do pó de folhas de M. esculenta Crantz, contendo ácido gálico e catequina, apresenta-se como uma alternativa promissora ao controle de S. frugiperda e de Atta sexdens rubropilosa.

Palavras-chave: Extrato vegetal, planta inseticida, compostos fenólicos, Spodoptera frugiperda, Atta sexdens

\begin{abstract}
The objective of this study was to characterize phenolic compounds and evaluate the effect, under laboratory conditions, of the cassava leaf powder methanol extract on the development of fall armyworm Spodoptera frugiperda and of leaf-cutting ant Atta sexdens rubropilosa. The extract was incorporated into an artificial diet, to which the armyworm was exposed, at concentrations of 250, 500, 1000 and $1500 \mathrm{mg}$ $\mathrm{kg}^{-1}$, in order to evaluate biological characteristics. Soon after the insects emergence, another experiment was conducted to verify the possible sub lethal activity of the extract; therefore, $S$. frugiperda couples were isolated in cages and eggs were collected and counted. The Manihot esculenta Crantz extract caused a reduction in the percentage of armyworm survival, as well as in the eggs number. Then, the same extract was solubilized in $10 \%$ ethanol and applied to ants; mortality was observed, compared to the control. It is possible to conclude that the M. esculenta Crantz leaf powder methanolic extract, containing gallic acid and catechin, is a promising alternative to control S. frugiperda and Atta sexdens rubropilosa.

Key words: Plant extract, plant insecticide, phenolic compounds, Spodoptera frugiperda, Atta sexdens
\end{abstract}

\footnotetext{
Químicos, Discentes de Doutorado, Dept ${ }^{\circ}$ de Química, Universidade Federal de Lavras, UFLA, Lavras, MG. E-mail: miriansantos.a@gmail.com; anapaula.quimica@hotmail.com; andersonbsbufla@yahoo.com.br

2 Profs. da UFLA, Lavras, MG. E-mail: angelita@dqi.ufla.br; adelir@dqi.ufla.br; gacarval@den.ufla.br

Bióloga, Discente de Doutorado do Dept ${ }^{\circ}$ de Entomologia, UFLA, Lavras, MG. E-mail: dejane_bio@yahoo.com.br

Eng $^{\mathrm{o}}$ Agr $^{\circ}$, Discente de Mestrado do Dept ${ }^{\circ}$ de Entomologia, UFLA, Lavras, MG. E-mail: rodrigo_lopes_oliveira@yahoo.com.br Autor para correspondência
} 


\section{Introdução}

Entre os inúmeros insetos pragas de culturas de importância econômica, destacam-se a lagarta militar ou lagarta-do-cartucho do milho, Spodoptera frugiperda (J.E. Smith) (Lepidoptera: Noctuidae) e a saúva Atta sexdens rubropilosa (Forel) (Hymenoptera: Formicidae). Ambas as pragas são polífagas e causam danos através da desfolha. A espécie S. frugiperda pode atacar culturas como milho, algodão, arroz, entre outras, provocando severos prejuízos devido à grande capacidade de desfolhamento que causa à parte aérea das plantas, ocasionando perdas que variam de 15 a $37 \%$, dependendo da cultura (BUSATO et al., 2005). As formigas causam perdas, como a morte de mudas, a redução do crescimento de árvores e provocam a diminuição da resistência da planta a outros insetos e agentes patogênicos à mesma. Estudos sobre os danos causados por formigas em florestas, mostraram que esses insetos são considerados pragas-severas podendo provocar perdas que podem chegar a 100\% (ZANETTI et al., 2003).

O controle dessas pragas vem sendo feito por meio da aplicação de inseticidas sintéticos, como piretróides e organofosforados (REDOAN et al., 2012; ZANETTI et al., 2008). Desta forma, são inúmeros os esforços na descoberta de novos produtos em alternativa aos pesticidas existentes e com isso a busca por princípios ativos de plantas contra insetos-praga tem se intensificado, já que, muitas vezes, o método de controle químico pode se mostrar ineficiente, além de causar uma série de efeitos adversos ao ambiente (GEORGES et al., 2008; AL-MAZRA'AWI; ATEYYAT, 2009; RAHUMAN et al., 2009).

Nesse contexto, é sabido que folhas de mandioca, Manihot esculenta Crantz, contém metabólitos secundários tais como polifenóis, lectinas e inibidores de tripsina (MELO et al., 2007). Entre as classes de metabólitos secundários de plantas com reconhecida atividade inseticida merecem destaque os compostos fenólicos, tais como lignina, flavonoides e taninos, conhecidos por conferirem proteção à planta contra a herbivoria (FRIEDMAN, 1997; MONTEIRO et al., 2005).

Assim, Cintra et al. (2002) constataram que insetos das ordens Lepidoptera, Orthoptera e Hymenoptera foram sensíveis à ingestão de compostos fenólicos em dietas artificiais. Neste contexto, Barbehenn et al. (2001) também verificaram que Orygia leucostogma Smith (Lepidoptera: Lymantiidae) e Helicoverpa zea Boddie (Lepidoptera: Noctuidae) tiveram maior deformidade de pupas e mortalidade de lagartas ao se alimentarem de fenóis originados do extrato de plantas da família Asteraceae, devido à oxidação de tecidos no intestino médio.

Não foram encontrados relatos de caracterização de compostos fenólicos nas folhas de mandioca, sendo que os estudos existentes relatam apenas o seu teor. Desta forma os objetivos neste trabalho foram caracterizar esses compostos e avaliar o efeito do extrato metanólico do pó de folhas de mandioca sobre o desenvolvimento da lagartado-cartucho $S$. frugiperda e da saúva-limão Atta sexdens rubropilosa.

\section{Material e Métodos}

Obtenção dos insetos

As lagartas de $S$. frugiperda utilizadas nos experimentos foram provenientes de criação de laboratório do Departamento de Entomologia da UFLA, em que as lagartas foram alimentadas com dieta artificial de Greene, Leppla e Dickerson (1976), e os adultos receberam como alimento solução aquosa de mel a $10 \%$. Para a montagem dos experimentos, foram utilizadas lagartas provenientes de segunda postura.

Para a execução do bioensaio com Atta sexdens rubropilosa, formigas foram coletadas em ninhos existentes no Campus da UFLA e levadas ao laboratório do Departamento de Entomologia.

\section{Coleta das folhas e preparo dos extratos}

Folhas maduras de mandioca (cultivar Pão da China), livres de pragas e doenças, obtidas junto ao 
Departamento de Agricultura, foram coletadas no período da manhã, no mês de abril, aos 12 meses de idade da planta e transportadas ao laboratório de Bioquímica da UFLA. As folhas foram lavadas em água destilada e, em seguida colocadas em estufas com circulação de ar para secagem por 48 $\mathrm{h}$, à temperatura entre 30 e $35^{\circ} \mathrm{C}$. Após secagem, as folhas tiveram seus pecíolos retirados e, em seguida, foram moídas para a obtenção de um pó com granulometria de 35 mesh. O teor de umidade foi determinado segundo AOAC (2005) nas folhas de mandioca e no pó obtido em triplicata.

Para o preparo dos extratos, o pó foi macerado em metanol durante $48 \mathrm{~h}$, na proporção de 10:15 $\left(\mathrm{p}^{-1}\right)$ e filtrado utilizando-se algodão hidrófilo. Ao resíduo foi adicionado mais metanol, sendo esse procedimento repetido sete vezes para garantir um bom rendimento da extração. As fases líquidas foram combinadas e concentradas em evaporador rotatório até a completa eliminação do solvente e, em seguida, foram liofilizadas, dando origem ao extrato metanólico (EM).

\section{Determinação de fenólicos no EM}

A dosagem de compostos fenólicos no EM foi determinada em triplicata, segundo metodologia da (AOAC, 2005), utilizando como padrão o ácido tânico.

\section{Caracterização dos compostos fenólicos}

As análises cromatográficas foram realizadas empregando o equipamento HPLC Agilent modelo 1100, equipado com uma bomba binária, injetor automático e detector com arranjo de diodos, no comprimento de onda $280 \mathrm{~nm}$. O EM e os padrões foram separados em uma coluna ascentis C18 25 $\mathrm{cm} \times 4,6 \mathrm{~mm}, 5 \mu \mathrm{m}$, conectada a uma pré-coluna ascentis C18 $2 \mathrm{~cm} \mathrm{x} \mathrm{4,0} \mathrm{mm,} 5 \mu \mathrm{m}$. A fase móvel foi composta pelas soluções: ácido acético 2\% (A) e metanol:água:ácido acético 70:28:2 $\left(\mathrm{v} \mathrm{v}^{-1} \mathrm{v}^{-1}\right)(\mathrm{B})$. As análises foram realizadas com tempo total de 50 minutos, a uma temperatura de $15^{\circ} \mathrm{C}$, em um sistema do tipo gradiente: $100 \%$ do solvente A de 0,01 a 5 minutos, $70 \%$ do solvente A de 5 a 25 minutos, $60 \%$ do solvente A de 25 a 43 minutos, $55 \%$ do solvente A de 43 a 50 minutos. O solvente A foi aumentado para $100 \%$ buscando manter o equilíbrio da coluna. O fluxo utilizado em todas as análises foi de $1 \mathrm{~mL}$ $\min ^{-1}$ e o volume de injeção foi de $20 \mu \mathrm{L}$.

Os padrões fenólicos utilizados foram: ácido gálico, galocatequina, 3,4-dihidroxidobenzeno, catequina, ácido clorogênico, epigalocatequina, ácido vanílico, epicatequina, ácido siríngico, ácido p-cumárico, ácido ferúlico, ácido m-cumárico, ácido o-cumárico, resveratrol, ácido elágico, ácido salicílico todos da marca Sigma-Aldrich (St. Louis, MO, EUA). As soluções estoque dos padrões foram preparadas em dimetil sufóxido e/ou metanol (Merck) em um intervalo de concentração de 0,10 a 177,15 mg L-1. O ácido acético e metanol (Merck, Darmstadt, Germany) foram usados na preparação da fase móvel e a água ultrapura foi obtida pelo sistema Milli-Q (Millipore, Billerica, MA, EUA).

$\mathrm{O} \mathrm{EM}$ e os padrões foram filtrados em uma membrana de nylon de $0,45 \mu \mathrm{m}$ (Millipore ${ }^{\circledR}$ ) e diretamente injetados no sistema cromatográfico. Cada solução foi injetada três vezes no sistema HPLC, com a finalidade de se obter a média das concentrações e dos tempos de retenção. Os compostos fenólicos no EM foram identificados por comparação com os tempos de retenção dos padrões. A quantificação foi realizada por meio da construção de curvas analíticas, no qual cada ponto representou a média de três repetições.

\section{Ensaio com lagartas de $S$. frugiperda}

O EM foi previamente solubilizado em água e incorporado à dieta artificial desenvolvida por Greene, Leppla e Dickerson (1976), nas concentrações de 250, 500, 1000 e $1500 \mathrm{mg} \mathrm{kg}^{-1}$. A incorporação do extrato foi realizada ao final do preparo da dieta, quando esta atingiu temperatura próxima a $55^{\circ} \mathrm{C}$, a fim de se evitar a degradação 
de compostos presentes no extrato. Os pedaços de dieta $(1,5 \mathrm{~cm}$ de diâmetro $\mathrm{x}$ 1,5 $\mathrm{cm}$ de altura) foram transferidos para recipientes plásticos de $50 \mathrm{~mL}$, onde foi inoculada uma lagarta de aproximadamente segundo instar. Na testemunha, os insetos receberam dieta acrescida de água. Para cada tratamento foram utilizados 72 insetos, cada um considerado uma repetição, sendo o experimento conduzido em delineamento inteiramente casualizado, com cinco tratamentos e setenta e duas repetições, cada parcela foi constituída por setenta e duas lagartas, mantidas de forma isolada. Os parâmetros avaliados foram: duração das fases larval e pupal, peso das pupas (após 24 horas de pupação), percentagem de sobrevivência dos insetos nas fases larval e pupal.

Logo após a emergência dos adultos, casais de $S$. frugiperda foram isolados em gaiolas, os insetos receberam como alimento solução aquosa de mel a $10 \%$. Nesse caso o experimento foi conduzido em delineamento inteiramente casualizado com cinco repetições por tratamento, sendo cada repetição constituída por um casal. Os experimentos foram realizados em sala climatizada a $25 \pm 2{ }^{\circ} \mathrm{C}$, umidade relativa de $70 \pm 10 \%$ e fotofase de 12 horas. A longevidade dos adultos foi determinada por meio de observações diárias. As posturas foram recolhidas diariamente para quantificação. Foram determinados os períodos de pré-oviposição, oviposição, longevidade dos adultos e número de ovos.

\section{Ensaio com formigas cortadeiras}

Para a execução do bioensaio com Atta sexdens rubropilosa, formigas, de diferentes castas, foram coletadas em ninhos existentes no Campus da UFLA e levadas ao laboratório do Departamento de Entomologia. Para a montagem dos testes, o EM foi solubilizado em etanol $10 \%$ e $1 \mu \mathrm{L}$ foi aplicado no protórax das formigas com o auxílio de microseringa, em quatro concentrações, sendo: 0,1 ; 0,$2 ; 0,3$ e $0,4 \mathrm{mg} \mathrm{mL}^{-1}$. Como testemunhas negativas utilizaram-se água e etanol 10\%. Para a aplicação dos extratos, as formigas foram imobilizadas com uso de $\mathrm{CO}_{2}$ por aproximadamente três segundos.
O delineamento do experimento foi inteiramente casualizado, sendo que cada parcela experimental foi representada por uma placa de Petri de $10 \mathrm{~cm}$ de diâmetro contendo 10 formigas e $10 \mathrm{~cm}^{3}$ de dieta artificial sólida confeccionada conforme metodologia de Bueno et al. (1997). O experimento foi realizado em câmara climatizada a $25 \pm 2{ }^{\circ} \mathrm{C}$, umidade relativa de $70 \pm 10 \%$ e fotofase de $12 \mathrm{~h}$. Avaliou-se o número de insetos mortos por um período de nove dias após as formigas receberem a aplicação dos extratos.

\section{Análises estatísticas}

Os parâmetros referentes a peso de pupa, sobrevivência na fase pupal, duração do período larval e pupal, pré-oviposição, oviposição, longevidade dos adultos e número de ovos, foram submetidos a “one way' ANOVA e quando significativas as médias dos tratamentos foram submetidas à análise de regressão $(p<0,05)$ em função da concentração do extrato (SAS, 2008).

Os dados associados à sobrevivência das lagartas durante a fase larval e às formigas durante $o$ período experimental foram submetidos à análise de sobrevivência, aplicando-se o modelo de Weibull, por meio do pacote Survival do software $R^{\circledR}$ (R DEVELOPMENT CORE TEAM, 2009). Após a seleção do modelo matemático mais adequado por meio da análise de resíduos, realizou-se a análise de contraste para verificar a semelhança entre os tratamentos empregados e a formação de grupos congêneres. Também foram calculados os tempos letais 50 (TL50), tempo necessário para matar 50\% dos insetos em teste, para cada grupo formado.

\section{Resultados e Discussão}

Compostos fenólicos

Os teores de umidade das folhas frescas e do pó das folhas de mandioca foram, em $\mathrm{g} 100 \mathrm{~g}^{-1}$, de 70,46 $\pm 0,07$ e $16,12 \pm 0,24$, respectivamente. 
O rendimento do EM do pó de folhas de mandioca foi cerca de $9,7 \%$, o que está de acordo com os resultados de Ayres et al. (2008) para o preparo de extratos a partir de outras espécies vegetais. O EM apresentou teor de compostos fenólicos de $1,58 \pm$

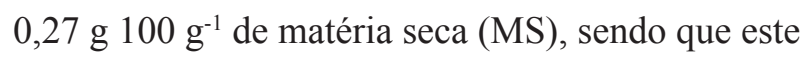
valor está abaixo da faixa de variação 4,72 $\pm 0,64$

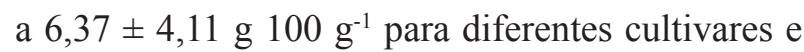
processamentos (CORRÊA et al., 2004; MELO et al., 2007).

No perfil cromatográfico do EM identificou-se os seguintes compostos fenólicos: ácido gálico $(35,00$ $\left.\pm 0,42 \mathrm{mg} 100 \mathrm{~g}^{-1}\right)$, catequina $(191,00 \pm 14,07 \mathrm{mg}$ $\left.100 \mathrm{~g}^{-1}\right)$. A respeito do modo de ação de compostos fenólicos sobre insetos, alguns autores relataram a formação de lesões degenerativas no intestino (ARTEEL; LINDROTH, 1992). Entretanto, mais recentemente, foi demonstrado que, para Malacosoma disstria Hübner (Lepidoptera: Lasiocampidae), a ingestão de compostos fenólicos não apenas produz um estresse oxidativo no conteúdo do lúmen do intestino médio, como também pode levar a um aumento do estresse oxidativo nos tecidos do intestino, o que provocaria a formação de lesões no mesmo (BARBEHENN et al., 2008).

\section{Ensaio com lagartas de S. frugiperda}

Lagartas alimentadas com dieta artificial contendo o EM nas concentrações de 500, 1000 e $1500 \mathrm{mg} \mathrm{kg}^{-1}$ apresentaram prolongamento da fase larval em comparação com a testemunha (Figura 1A) Referente à fase de pupa, somente os tratamentos contendo 1000 e $1500 \mathrm{mg} \mathrm{kg}^{-1}$ do referido extrato alongaram esta fase em comparação ao controle (Figura 1B). O prolongamento da fase jovem de insetos causado pela ingestão de produtos de origem natural já foi relatado na literatura. Nomura e Itioca (2002) observaram o aumento na duração das fases larval e pupal, a partir da aplicação de taninos sintéticos em dietas artificiais de Spodoptera litura (Fabricius) (Lepidoptera: Noctuidae), e constataram ainda que a redução de crescimento das larvas foi proporcional à concentração de substância ingerida. Tirelli et al. (2010) também observaram o prolongamento das fases larval e pupal de $S$. frugiperda quando estudaram a fração tânica de diversas cascas de árvore. De forma análoga, Santiago et al. (2008) verificaram que o extrato de Ricinus comunis L. (Euforbiaceae) provocou alongamento na fase larval de $S$. frugiperda. O prolongamento do período larval dessa praga, em consequência do tratamento com extratos de Trichilia pallida, também foi observado por (THOMAZINI; VENDRAMIM; LOPES, 2000; ROEL, 2001). Pode-se dizer que a interferência do extrato empregado foi menor na duração da fase pupal quando comparada com a fase larval. De forma análoga Rodriguez e Vendramim (1996) também observaram que o estágio pupal de $S$. frugiperda foi menos afetado pelos extratos de diversas plantas quando incorporados em dieta artificial, em comparação com o estágio larval.

O alongamento das fases larval e pupal observado possivelmente ocorreu devido a presença de substâncias fenólicas, tóxicas ao inseto, como por exemplo o ácido gálico, o qual foi identificado no extrato empregado nesse experimento. Segundo os estudos de Urrea-Bulla, Suárez e Moreno-Murillo (2004) esse tanino age como fagodeterrente contra larvas de $S$. frugiperda. Uma maior duração da fase larval, em campo, é uma vantagem já que aumenta a exposição aos fatores de mortalidade natural tais como predadores e parasitoides.

Foi constatadoquetodas as concentrações testadas reduziram a sobrevivência dos insetos durante a fase larval, em comparação com a testemunha. O EM foi capaz de provocar mortalidade acima de $40 \%$ após o término do período de avaliação, com TL50 de 30,02 dias (Figura 2). 
Figura 1. Efeito do extrato metanólico (EM) do pó de folhas de mandioca, em diferentes concentrações, sobre duração da fase larval (A), duração da fase pupal (B) e número de ovos (C).
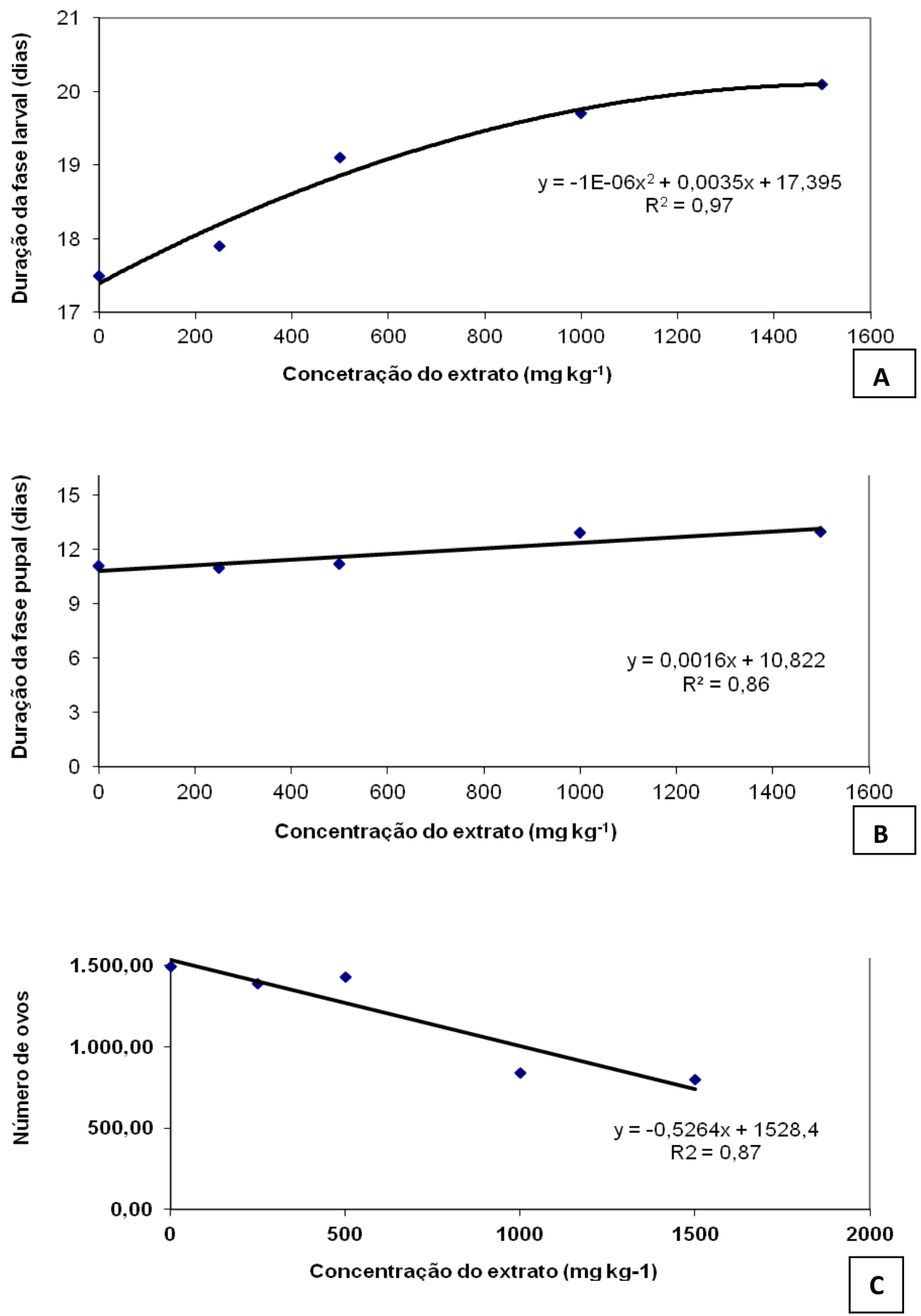

Fonte: Elaboração dos autores. 
Figura 2. Sobrevivência de lagartas de Spodoptera frugiperda, ao longo do tempo, alimentadas com dieta artificial contendo extrato metanólico (EM) de pó de folhas de mandioca em diferentes concentrações, sendo y $=\exp \left((\mu)^{\wedge}(-\right.$ $\alpha)^{*}\left(\mathrm{x}^{\wedge} \alpha\right)$, em que $\mathrm{y}=$ sobrevivência; $\mu=$ tempo letal $50 ; \alpha=1,003917$ e $\mathrm{x}=$ tempo (dias).

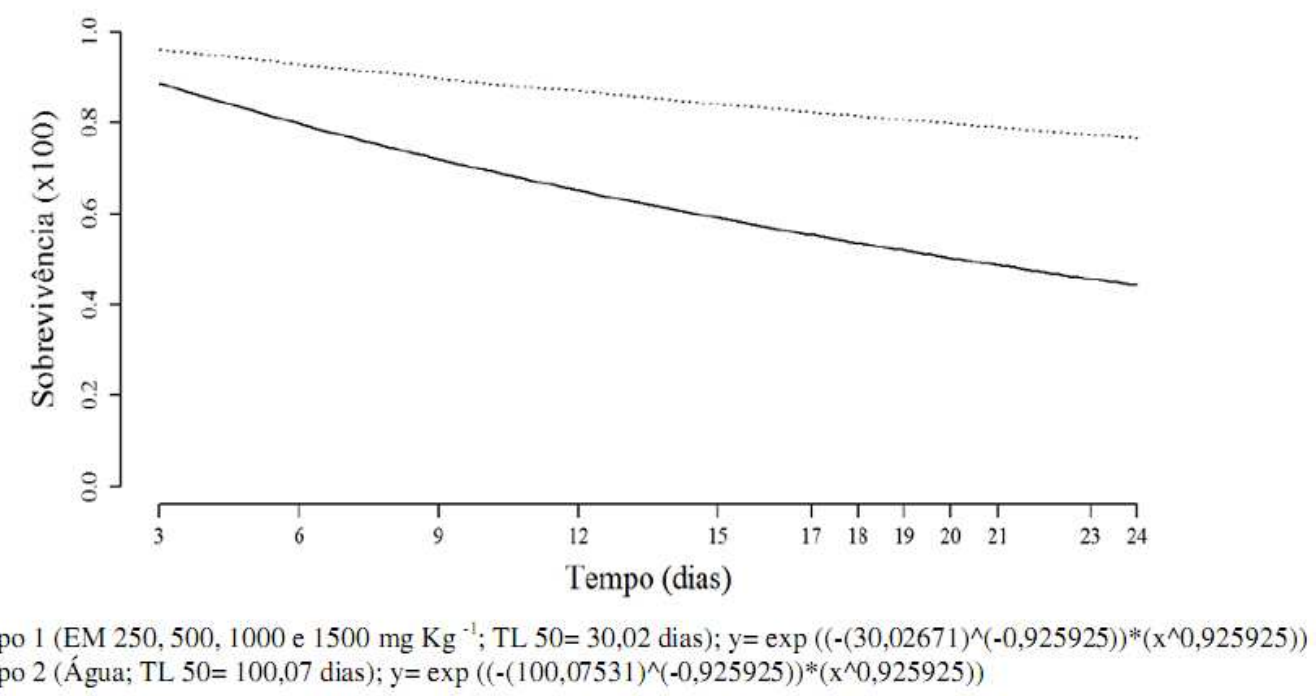

Fonte: Elaboração dos autores.

De forma semelhante ao constatado nesse trabalho, o aumento na duração das fases larval e pupal e acréscimo de mortalidade na fase larval também foram relatados por Tandon, Mittal e Pant (2008), quando aplicaram óleos essenciais de Vitex trifolia e $V$. agnus-castus L. (Verbenaceae) em lagartas de Spilosoma obliqua Walker (Lepidoptera: Arctiidae). Entretanto, para ambos os tratamentos, não foram observadas relações entre as doses utilizadas e a mortalidade acumulada, já que todos os tratamentos foram agrupados em um único grupo na distribuição de Weibull. Em estudo utilizando óleo essencial de Pilocarpus spicatus Saint-Hilaire (Rutaceae) para o controle de ninfas de quinto instar de Rhodnius prolixus Stål (Hemiptera: Reduviidae), de maneira semelhante ao constatado no presente trabalho, os autores não puderam propor uma relação entre a dose aplicada e seu efeito na mortalidade (MELLO et al., 2007).

No que diz respeito ao peso de pupas e à sobrevivência na fase pupal, pode-se dizer que o extrato não afetou esses parâmetros, já que não houve diferença estatística entre os tratamentos e a testemunha (Tabela 1). Torrecillas e Vendramim
(2001) observaram que as alterações no desenvolvimento de $S$. frugiperda ocorreram apenas na fase larval; isto pode ser explicado pelo fato de ser essa fase na qual o inseto se alimenta, o que o torna mais exposto aos possíveis aleloquímicos presentes no extrato da planta inseticida.

A duração dos períodos de pré-oviposição, oviposição e a longevidade dos adultos (tanto machos quanto fêmeas) não foram afetadas por nenhuma das concentrações do EM testadas (Tabela 2).

Em relação ao número de ovos, constatou-se que os tratamentos com as concentrações mais elevadas, com 1000 e $1500 \mathrm{mg} \mathrm{kg}^{-1}$, apresentaram valores inferiores de número de ovos, diferindo estatisticamente da testemunha. Verificou-se assim, uma redução na oviposição (Figura 1C), quando as larvas de Spodoptera frugiperda ingeriram o EM adicionado à dieta artificial. Para Costa, Silva e Fiuza (2004), a redução do número de ovos e a inibição da oviposição são importantes efeitos dos extratos vegetais sobre a reprodução dos insetos, sendo que a ocorrência de esterilidade está geralmente associada a distúrbios alimentares e deficiência nutricional. 
Levando-se em consideração que a catequina foi identificada no EM, acredita-se que esse composto possa ser um dos responsáveis pelo efeito do extrato sobre $S$. frugiperda, pois segundo Monteiro et al. (2005) a catequina apresenta maior toxicidade para os insetos que os taninos.

Tabela 1. Efeito do extrato metanólico (EM) do pó de folhas de mandioca, em diferentes concentrações, sobre o desenvolvimento de Spodoptera frugiperda.

\begin{tabular}{lcc}
\hline $\begin{array}{l}\text { Tratamento } \\
\left(\mathrm{mg} \mathrm{kg}^{-1}\right)\end{array}$ & $\begin{array}{c}\text { Peso de pupas } \\
(\mathrm{mg})\end{array}$ & $\begin{array}{c}\text { Sobrevivência na fase pupal }{ }^{\text {ns }} \\
(\%)\end{array}$ \\
\hline EM 250 & $260,0 \pm 1,75$ & $97,2 \pm 7,30$ \\
EM 500 & $260,0 \pm 2,70$ & $96,3 \pm 5,43$ \\
EM 1000 & $270,0 \pm 3,10$ & $93,9 \pm 1,78$ \\
EM 1500 & $270,0 \pm 5,30$ & $91,7 \pm 2,77$ \\
Água & $260,0 \pm 3,30$ & $88,9 \pm 3,23$ \\
\hline F & 1,8753 & 1,1573 \\
P & 0,2791 & 0,3721 \\
\hline
\end{tabular}

${ }^{n s}$ Médias não significativas pelo teste One-way anova a 5\% de probabilidade.

Fonte: Elaboração dos autores.

Tabela 2. Efeito do extrato metanólico (EM) do pó de folhas de mandioca em diferentes concentrações, sobre os períodos de pré-oviposição e oviposição, longevidade dos adultos e número de ovos de Spodoptera frugiperda.

\begin{tabular}{|c|c|c|c|c|}
\hline \multirow[t]{2}{*}{$\begin{array}{c}\text { Tratamento } \\
\left(\mathrm{mg} \mathrm{kg}^{-1}\right)\end{array}$} & \multirow[t]{2}{*}{$\begin{array}{l}\text { Período de pré- } \\
\text { oviposição } \\
\text { (dias) }\end{array}$} & \multirow[t]{2}{*}{$\begin{array}{c}\text { Período de } \\
\text { oviposiçãão }^{\text {ns }} \\
\text { (dias) }^{\text {dias }}\end{array}$} & \multicolumn{2}{|c|}{$\begin{array}{c}\text { Longevidades }^{\mathrm{ns}} \\
\text { (dias) }\end{array}$} \\
\hline & & & femeas & Machos \\
\hline EM 250 & $4,50 \pm 0,28$ & $5,70 \pm 0,67$ & $11,20 \pm 0,69$ & $11,80 \pm 2,28$ \\
\hline EM 500 & $3,90 \pm 0,42$ & $6,50 \pm 0,61$ & $11,30 \pm 0,70$ & $13,70 \pm 1,15$ \\
\hline EM 1000 & $4,30 \pm 0,19$ & $5,70 \pm 0,88$ & $11,80 \pm 0,85$ & $11,20 \pm 1,09$ \\
\hline EM 1500 & $4,30 \pm 0,19$ & $5,50 \pm 1,07$ & $8,00 \pm 0,73$ & $13,30 \pm 2,14$ \\
\hline Água & $3,50 \pm 0,34$ & $8,10 \pm 1,19$ & $11,20 \pm 1,27$ & $12,60 \pm 0,82$ \\
\hline $\mathrm{F}$ & 0,7341 & 0,7573 & 1,6747 & 1,3700 \\
\hline $\mathrm{P} \leq$ & 0,5713 & 0,5310 & 0,1791 & 0,2753 \\
\hline
\end{tabular}

${ }^{n s}$ Médias não significativas pelo teste One-way anova a 5\% de probabilidade.

Fonte: Elaboração dos autores.

\section{Ensaio realizado com formigas}

$\mathrm{Na}$ Figura 3 é mostrada a análise de sobrevivência das formigas Atta sexdens rubropilosa quando expostas a aplicação tópica de diferentes concentrações do EM. De acordo com os resultados, pôde-se observar que de modo geral, o EM apresentou efeito negativo sobre a sobrevivência das formigas ao longo do tempo, verificando-se redução no tempo de sobrevivência dos insetos tratados com o extrato proporcional ao aumento da concentração do mesmo, com médias de tempo letal (TL50) estimadas em 6,54; 7,60 e 9,17 dias nos grupos 1, 2 e 3, respectivamente. Com relação aos tratamentos testemunha, constatou-se maior média de TL50 (17,22 dias) quando houve aplicação tópica de água sobre as formigas, sendo seguida pela média do tratamento que empregou etanol 10\% (14,31 dias). 
Existem relatos na literatura de que muitas plantas demonstram ser potencialmente tóxicas a esses insetos, como exemplo pode-se citar o trabalho de Hebling et al. (2000), que observaram alterações metabólicas nas formigas retiradas de formigueiros tratados com folhas de gergelim e Morini et al. (2005), demonstraram que as ligninas isoladas do óleo de gergelim são tóxicas para formigas. Neste trabalho vereficou-se que o EM mostrou-se eficiente no controle de Atta sexdens rubropilosa.

Figura 3. Sobrevivência de Atta sexdens tratadas topicamente com diferentes concentrações de extrato metanólico (EM) de pó de folhas de mandioca em diferentes concentrações, sendo $y=\exp \left((\mu)^{\wedge}(-\alpha)^{*}\left(\mathrm{x}^{\wedge} \alpha\right)\right.$, em que $\mathrm{y}=$ sobrevivência; $\mu=$ tempo letal 50; $\alpha=2,735197$ e $\mathrm{x}=$ tempo (dias).

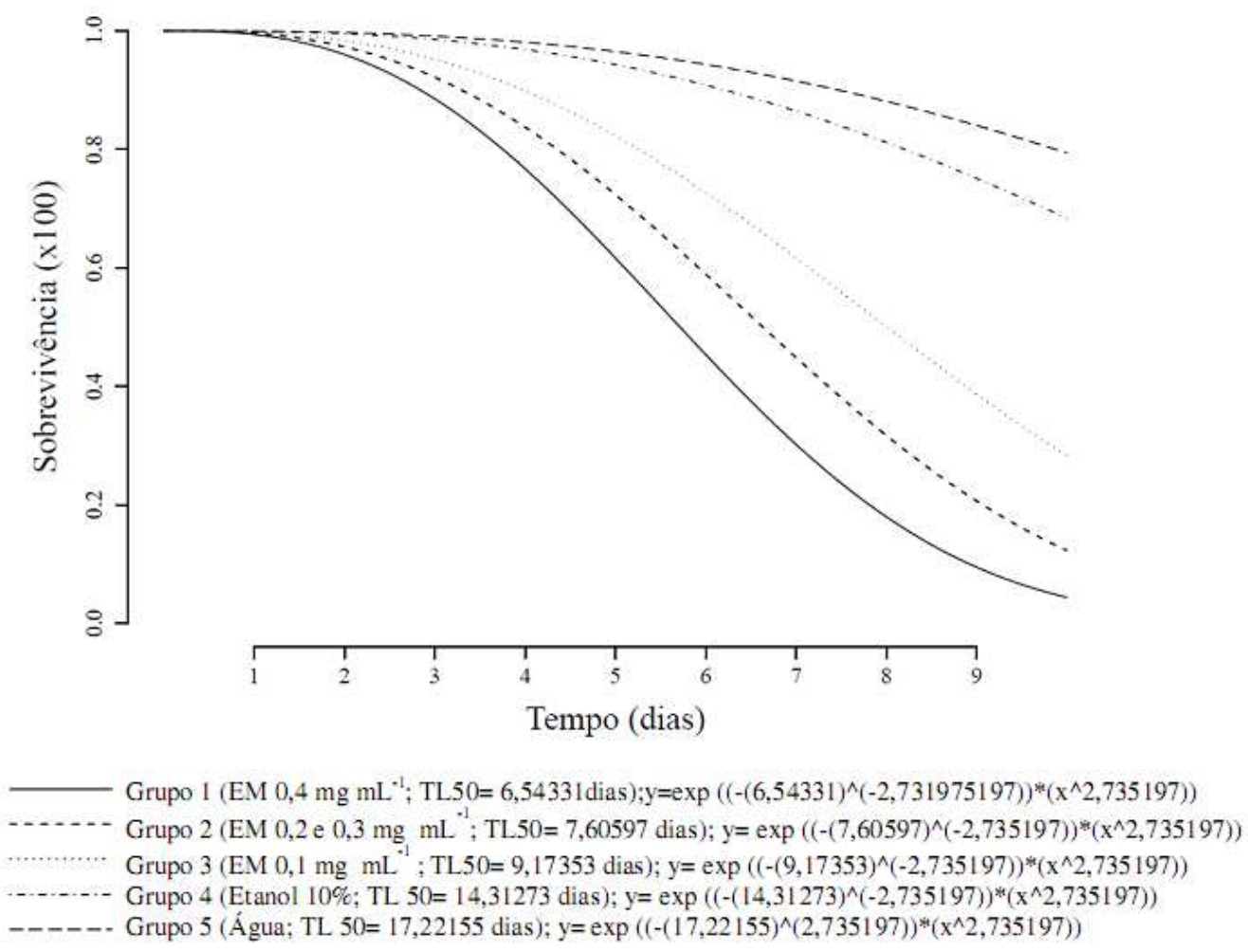

Fonte: Elaboração dos autores.

\section{Conclusões}

Conclui-se que o EM das folhas de $M$. esculenta Crantz foi capaz de provocar efeito subletal aumentando o tempo necessário para o desenvolvimento de $S$ frugiperda prolongando as fases larval e pupal, além de provocar redução no número de ovos, sendo que a redução na sobrevivência da lagarta e de Atta sexdens rubropilosa também foi observada ao longo do tempo.
Foram identificados no extrato ácido gálico e catequina que são tóxicos e podem causar efeitos nocivos aos insetos, de modo que a atividade encontrada foi atribuída à presença desses compostos fenólicos.

\section{Agradecimentos}

A FAPEMIG pelo apoio financeiro e à CAPES pela concessão da bolsa de doutorado ao primeiro autor. 


\section{Referências}

AL-MAZRA'AWI, M. S.; ATEYYAT, M. Insecticidal and repellent activities of medicinal plant extracts against the sweet potato whitefly, Bemisia tabaci (Hom.: Aleyrodidae) and its parasitoid Eretmocerusmundus (Hym.: Aphelinidae). Journal of Pest Science, Berlin, v. 82, n. 2, p. 149-154, 2009.

ARTEEL, G. E.; LINDROTH, R. L. Effects of aspen phenolic glycosides on gypsy moth (Lepidoptera: Lymantriidae) susceptibility to Bacillus thuringiensis. The Great Lakes Entomologist, Michigan, v. 25, n. 4, p. 239-244, 1992.

ASSOCIATION OF OFFICIAL ANALYTICAL CHEMISTS - AOAC. Official methods of analysis of the association of the analytical chemists. 18. ed. Maryland: AOAC, 2005.

AYRES, M. C. C.; BRANDÃO, M. S.; VIEIRAJÚNIOR, G. M.; MENOR, J. C. A. S.; SILVA, H. B.; SOARES, M. J. S.; CHAVES, M. H. Atividade antibacteriana de plantas úteis e constituintes químicos da raiz de Coperniciaprunifera. Revista Brasileira de farmacognosia, Maringá, v. 1, n. 18, p. 90-97, 2008.

BARBEHENN, R. V.; BUMGARNER, S. L.; ROOSEN, E. F.; MARTIN, M. M. Antioxidant defenses in caterpillars: role of the ascorbaterecycling system in the midgut lumen. Journal of Insect Physiology, Oxford, v. 47, n. 4-5, p. 349-357, 2001.

BARBEHENN, R. V.; MABEN, R. E.; KNOESTER, J. J. Linking phenolic oxidation in the midgut lumen with oxidative stress in the midgut tissues of a treefeeding caterpillar Malacosoma disstria (Lepidoptera: Lasiocampidae). Environmental Entomology, Lanham, v. 37, n. 5, p. 1113-1118, 2008.

BUENO, O. C.; MORINI, M. S. C.; PAGNOCCA, F. C.; HEBLING, M. J. A.; SILVA, O. A. Sobrevivência de operárias de Atta sexdens rubropilosa Forel (Hymenoptera: Formicidae) isoladas do formigueiro e alimentadas com dietas artificiais. Anais da Sociedade Entomológica do Brasil, Londrina, v. 26, n. 1, p. 107113, 1997.

BUSATO, G. R.; GRUTZMACHER, A. D.; GARCIA, M. S.; GIOLO, F. P.; ZOTTI, M. J.; STEFANELLO, JUNIOR, G. J. Biologia comparada de populações de Spodoptera frugiperda (J.E Smith) (Lepidoptera: Noctuidae) em folhas de milho e arroz. Neotropical Entomology, Londrina, v. 34, n. 5, p. 743-750, 2005.
CINTRA, P.; MALASPINA, O.; PETACCI, F.; FERNANDES, J. B.; BUENO, O. C.; VIEIRA, P. C.; SILVA, M. F. G. F. Toxicity of Dimorphandra mollis to workers of Apis mellifera. Journal of the Brazilian Chemical Society, Campinas, v. 13, n. 1, p. 115-118, 2002.

CORREAA, A. D.; SANTOS, S. R. dos; ABREU, C. M. P. de; JOKL, L.; SANTOS, C. D. dos. Remoção de polifenóis da farinha de folhas de mandioca. Ciência e Tecnologia de Alimentos, Campinas, v. 24, n. 2, p. 159164, 2004.

COSTA, E. L. N.; SILVA, R. F. P. da; FIUZA, L. M. Efeitos, aplicações e limitações de extratos de plantas inseticidas. Acta Biologica Leopoldensia, São Leopoldo, v. 26, n. 2, p. 173-85, 2004.

FRIEDMAN, M. Chemistry, biochemistry, and dietary role of potato polyphenols. A review. Journal of Agriculture and Food Chemistry, Easton, v. 45, n. 5, p. 1523-1540, 1997.

GEORGES, K.; JAYAPRAKASAM, B.; DALAVOY, S. S.; NAIR, M. G. Pest-managing activities of plant extracts and anthraquinones from Cassia nigricans from Burkina Faso. Bioresource Technology, Essex, v. 99, n. 6, p. 2037-2045, 2008.

GREENE, G. L.; LEPPLA, N. C.; DICKERSON, W. A. Velvetbean caterpillar: a rearing procedure and artificial medium. Journal of Economic Entomology, Lanham, v. 69, n. 4, p. 487-488, 1976.

HEBLING, M. J. A.; BUENO, O. C.; PAGNOCCA, F. C.; SILVA, O. A. da; MAROTI, P. S. Toxic effects of Canavalia ensiformis L. (Leguminosae) on laboratory colonies of Atta sexdens L. (Hym. Formicidae). Journal of Applied Entomology, Berlin, v. 124, n. 1, p. 33-35, 2000.

MELLO, C. B.; UZEDA, C. D.; BERNARDINO, M. V.; MENDONÇA-LOPES, D. J. A. F. P. C. A.; GUERRA, M. S.; OLIVEIRA, A. P.; ROCHA, L. M.; GONZALES, M. S. Effects of the essential oil obtained from Pilocarpusspicatus Saint-Hilaire (Rutaceae) on the development of Rhodniusprolixusnymphae. Revista Brasileira de Farmacognosia, Maringá, v. 17, n. 4, p. 514-520, 2007.

MELO, D. S. de; CORRÊA, A. D.; MARCOS, F. C. A.; SOUZA, R. V. de; ABREU, C. M. P. de; SANTOS, C. D. dos. Efeito da farinha de folhas de mandioca sobre a peroxidação lipídica, o perfil lipídico sanguíneo e o peso de fígado de ratos. Ciência e Agrotecnologia, Lavras, v. 31, n. 2, p. 420-428, 2007. 
MONTEIRO, J. M.; ALBUQUERQUE, U. P.; ARAÚJO, E. L.; AMORIM, E. L. C. Taninos: uma abordagem da química à ecologia. Química Nova, São Paulo, v. 28, n. 5, p. 892-896, 2005.

MORINI, M. S. C.; BUENO, O. C.; BUENO, F. C.; LEITE, A. C.; HEBLING, M. J. A.; PAGNOCCA, F. C.; FERNANDES, J. B.; VIEIRA, P. C.; SILVA, M. F. G. F. da. Toxicity of sesame seed to leafcutting ant Atta sexdens rubropilosa (Hymenoptera: Formicidae). Sociobiology, Boston, v. 45, n. 1, p. 195-204, 2005.

NOMURA, M.; ITIOKA, T. Effects of synthesized tannin on the growth and survival of a generalist herbivorous insect, the common cutworm, Spodoptera litura Fabricius (Lepidoptera: Noctuidae). Applied Entomology and Zoology, Tokyo, v. 37, n. 2, p. 285-289, 2002.

R DEVELOPMENT CORE TEAM. $R$ : a language and environment for statistical computing. Vienna: $R$ Foundation for Statistical Computing, 2009. Disponível em: <http://www.R-project.org>. Acesso em: 17 jul. 2012.

RAHUMAN, A. A.; BAGAVAN, A.; KAMARAJ, C.; VADIVELU, M.; ABDUZ-ZAHIR, A.; ELANGO, G.; PANDIYAN, G. Evaluation of indigenous plant extracts against larvae of Culex quinquefasciatus Say (Diptera: Culicidae). Journal Parasitology Research, Berlin, v. 104, n. 3, p. 637-643, 2009.

REDOAN, A. C. M.; CARVALHO, G. A.; CRUZ, I.; FIGUEIREDO, M. de L. C.; SILVA, R. B. da. Seletividade de inseticidas utilizados no controle de spodoptera Frugiperda (J.E. Smith) (Lepdoptera: Noctuidae) para ovos e ninfas de Doru luteipes (Scudder) (Dermaptera: Forficulidae). Revista Brasieira de Milho e Sorgo, Sete Lagoas, v. 11, n. 1, p. 25-34, 2012.

RODRIGUEZ, H. C.; VENDRAMIM, J. D. Toxicidade de extratos aquosos de Meliaceae em Spodoptera frugiperda (Lepidoptera: Noctuidae). Manejo Integrado de Pragas, Turrialba, San José, v. 42, n. 1, p. 14-22, 1996.

ROEL, A. R. Utilização de plantas com propriedades inseticidas: uma contribuição para o Desenvolvimento Rural Sustentável. Revista Internacional de Desenvolvimento, Campo Grande, v. 1, n. 2, p. 43-50, 2001.
SANTIAGO, G. P.; PÁDUA, L. E. M.; SILVA, P. R. R.; CARVALHO, E. M. S.; MAIA, C. B. Efeitos de Extratos de Plantas na Biologia de Spodoptera frugiperda (J. E. Smith 1797) (Lepidoptera: Noctuidae) Mantida em dieta Artificial. Ciência e Agrotecnologia, Lavras, v. 32, n. 3, p. 792-796, 2008.

STATISTIC ANALYSIS SYSTEM - SAS. Institute, SAS/STAT user's guide v.8. Cary, NC, USA: SAS Institute. 2008.

TANDON, S.; MITTAL, A. K.; PANT, A. K. Insect growth regulatory activity of Vitex trifolia and Vitex agnuscastus essential oils against Spilosoma oblique. Fitoterapia, Milano, v. 79, n. 4, p. 283-286, 2008.

THOMAZINI, A. P. B. W.; VENDRAMIM, J. D.; LOPES, M. T. R. Extratos aquosos de Trichilia pallida e a traça-do-tomateiro. Scientia Agricola, Piracicaba, v. 57, n. 1, p. 13-17, 2000.

TIRELli, A. A.; ALVES, D. S.; CARVALHO, G. A.; SÂMIA, R. R.; BRUM, S. S.; GUERREIRO, M. C. Efeito de frações tânicas sobre parâmetros biológicos e nutricionais de Spodoptera frugiperda (Lepidoptera: Noctuidae). Ciência e Agrotecnologia, Lavras, v. 34, n. 6, p. 1417-1424, 2010.

TORRECILLAS, S. M.; VENDRAMIM, J. D. Extrato aquoso de ramos Trichila pallida e o desenvolvimento de Spodoptera frugiperda em genótipos de milho. Scientia Agrícola, Piracicaba, v. 58, n. 1, p. 27-31, 2001.

URREA-BULLA, A.; SUÁREZ, M. M.; MORENOMURILLO, B. Biological activity of phenolic compounds from Alchornea glandulosa. Fitoterapia, Hefei, v. 75, n. 3, p. 392-394, 2004.

ZANETTI, R.; JANUCIO, J. C.; VILELA, E. F.; LEITE, H. G.; JAFFÉ, K.; OLIVEIRA, A. C. Level of economic damage for leaf-cutting ants (Hymenoptera: Formicidae) in Eucalyptus plantations in Brazil. Sociobiology, Feira de Santana, v. 42, n. 2, p. 442-443, 2003.

ZANETTI, R.; ZANUCIO, J, C.; SOUZA-SILVA, A.; MENDONÇA, L. A.; MATTOS, J. O. S.; RIZENTAL, M. $\mathrm{S}$. Eficiência de produtos termonebulígenos no controle de Atta laevigata (Himenoptera: Formicidae) em plantio de eucalipto. Ciência e Agrotecnologia, Lavras, v. 32, n. 4, p. 1313-1316, 2008. 
